\title{
THERMAL FLUID COUPLED ANALYSIS OF HYDROTHERMAL DESTRUCTION REACTOR
}

\section{TOKIHIRO SUGIMOTO ${ }^{1}$, HIROYUKI KURAMAE ${ }^{2}$, MASAHIDE MATSUMOTO ${ }^{3}$ AND NOBUHISA WATANABE ${ }^{4}$}

\author{
${ }^{1}$ Graduate School of Osaka Institute of Technology \\ 1-45 Chayamachi, Kita-ku, Osaka 530-8568, Japan \\ m1m20r14@st.oit.ac.jp, http://www.oit.ac.jp/ \\ ${ }^{2}$ Osaka Institute of Technology \\ 1-45 Chayamachi, Kita-ku, Osaka 530-8568, Japan \\ hiroyuki.kuramae@oit.ac.jp, http://www.oit.ac.jp/ \\ ${ }^{3}$ Osaka Institute of Technology \\ 5-1-16 Omiya, Asahi-ku, Osaka 535-8585, Japan \\ masahide.matsumoto@oit.ac.jp, http://www.oit.ac.jp/ \\ ${ }^{4}$ Osaka Institute of Technology \\ 5-1-16 Omiya, Asahi-ku, Osaka 535-8585, Japan \\ nobuhisa.watanabe@oit.ac.jp, http://www.oit.ac.jp/
}

Key words: Polychlorinated Biphenyl, Hydrothermal Oxidative Destruction Reactor, Thermal Fluid Analysis, OpenFOAM

\begin{abstract}
Multi-regionally coupled analysis of thermal fluid flow and heat conduction of solid using OpenFOAM is carried out to clarify the behavior of hydrothermal oxidative destruction reactor of polychlorinated biphenyls (PCBs). Internal fluid of the reactor assumes a single-phase hot water without chemical reactions considering temperature dependence of thermophysical properties. Compressible Navier-Stokes equation with buoyancy force and energy equation with gravity term are alternately solved for the thermal fluid analysis. In order to consider conjugate heat transfer between the internal fluid and the reactor vessel, two-regionally coupled analysis of the fluid and vessel was executed by chtMultiRegionFoam solver in the OpenFOAM. To verify coupling effect, the multi-regionally coupled analyses results were compared with thermal fluid analysis of the internal fluid or heat conduction analysis of the vessel.
\end{abstract}

\section{INTRODUCTION}

Hydrothermal oxidative destruction reactor decomposes polychlorinated biphenyls (PCBs, $\mathrm{C}_{12} \mathrm{H}_{(10-n)} \mathrm{Cl}_{n}$ ) into water $\left(\mathrm{H}_{2} \mathrm{O}\right)$, carbon dioxide $\left(\mathrm{CO}_{2}\right)$ and sodium chloride $(\mathrm{NaCl})$ by dechlorination with sodium carbonate $\left(\mathrm{Na}_{2} \mathrm{CO}_{3}\right)$ and oxidative decomposition with liquid oxygen $\left(\mathrm{O}_{2}\right)$ under high temperature $370{ }^{\circ} \mathrm{C}$ and high pressure 26.5 MPa [1]. In these reactor vessels, wall thinning due to corrosion was observed on bottom inner wall. At present, the reactors have been safely maintained and operated by adding a bottom 
partition to prevent chemical sinking and supplying hot water to the reactor vessel bottom to control the temperature. Thermal fluid analysis of the hydrothermal oxidative destruction reactors is necessary to clarify the corrosion mechanism.

A finite volume analysis (FVA) code OpenFOAM [2] is used to perform a coupled analysis of the internal fluid flow and heat conduction in the reactor vessel with multi-region considering the conjugate heat transfer on the solid-liquid interface. This paper describes verification of the multi-regionally coupled analysis by comparing with single region analysis, which is thermal fluid analysis of the internal fluid or heat conduction analysis of the vessel body.

\section{METHOD OF THE ANALYSIS}

\subsection{Governing equations}

In this study, since chemical reactions in hydrothermal oxidative decomposition of PCBs are not considered, the internal fluid in the vessel assumes a single hot water. To analyze steady state of thermal fuid behavior in the vessel, the continuous equation, the compressible Navier-Stokes equations with buoyancy force, and the energy equation per unit mass with gravity terms are as follows:
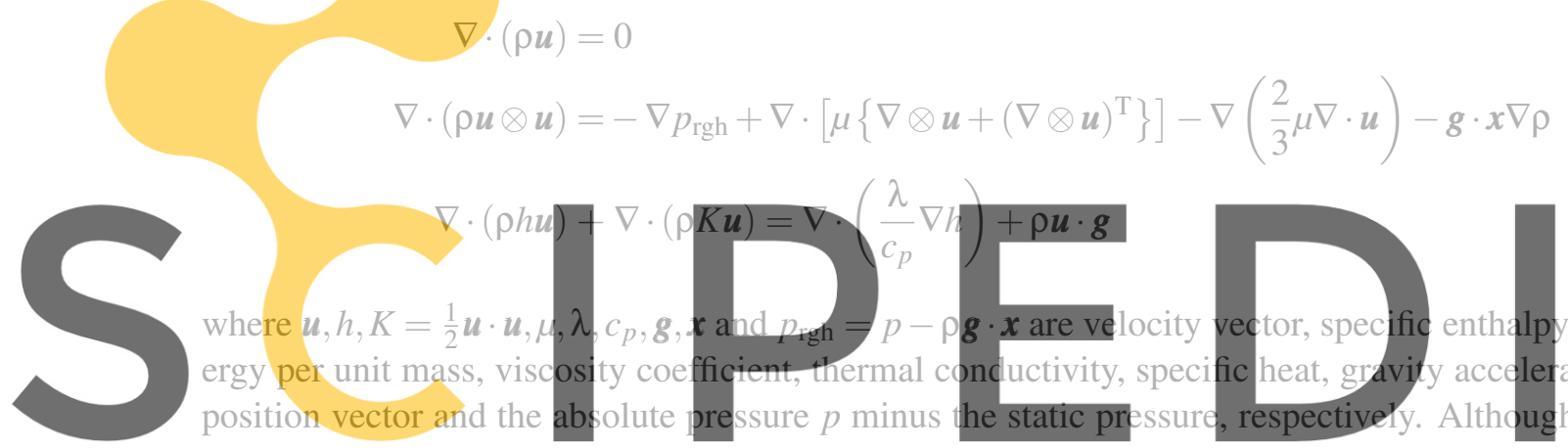

$\nabla \cdot(\rho h \boldsymbol{u})+\nabla \cdot(\rho \boldsymbol{K} \boldsymbol{u})=\boldsymbol{\nabla} \cdot\left(\frac{\lambda}{c_{p}} \nabla h\right.$
where $\boldsymbol{u}, h, K=\frac{1}{2} \boldsymbol{u} \cdot \boldsymbol{u}, \boldsymbol{\mu}, \lambda, c_{p}, g, \boldsymbol{x}$ and $\left.p_{\boldsymbol{r}}\right)=p-\rho$
ergy per unit mass, viscosity coefficient, thermal con
position vector and the absolute pressure $p$ minus the


equation (3) does not include heat generation term, the heat of chemical reaction is considered by a

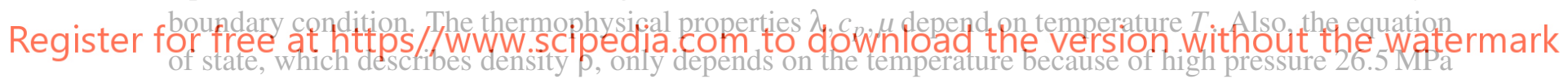

in the vessel. The turbulence is considered using the standard $k-\varepsilon$ model.

The heat conduction equation without heat generation term for the reactor vessel body is as follow:

$$
\nabla \cdot\left(\frac{\lambda}{\rho c_{p}} \nabla h\right)=0
$$

where the thermophysical properties of the vessel body $\lambda, \rho, c_{p}$ are assumed to be constant without considering the temperature dependence.

\subsection{Method of the coupled analysis using OpenFOAM}

In the coupled analysis of heat transfer between the internal fluid and the vessel body (solid), the continuities of temperature $T$ and heat flux $(-\lambda \nabla T)$ are satisfied on the solid-liquid interface as follows:

$$
\begin{aligned}
T_{\text {solid }} & =T_{\text {fluid }} \\
\lambda_{\text {solid }} \nabla T_{\text {solid }} & =\lambda_{\text {fluid }} \nabla T_{\text {fluid }}
\end{aligned}
$$




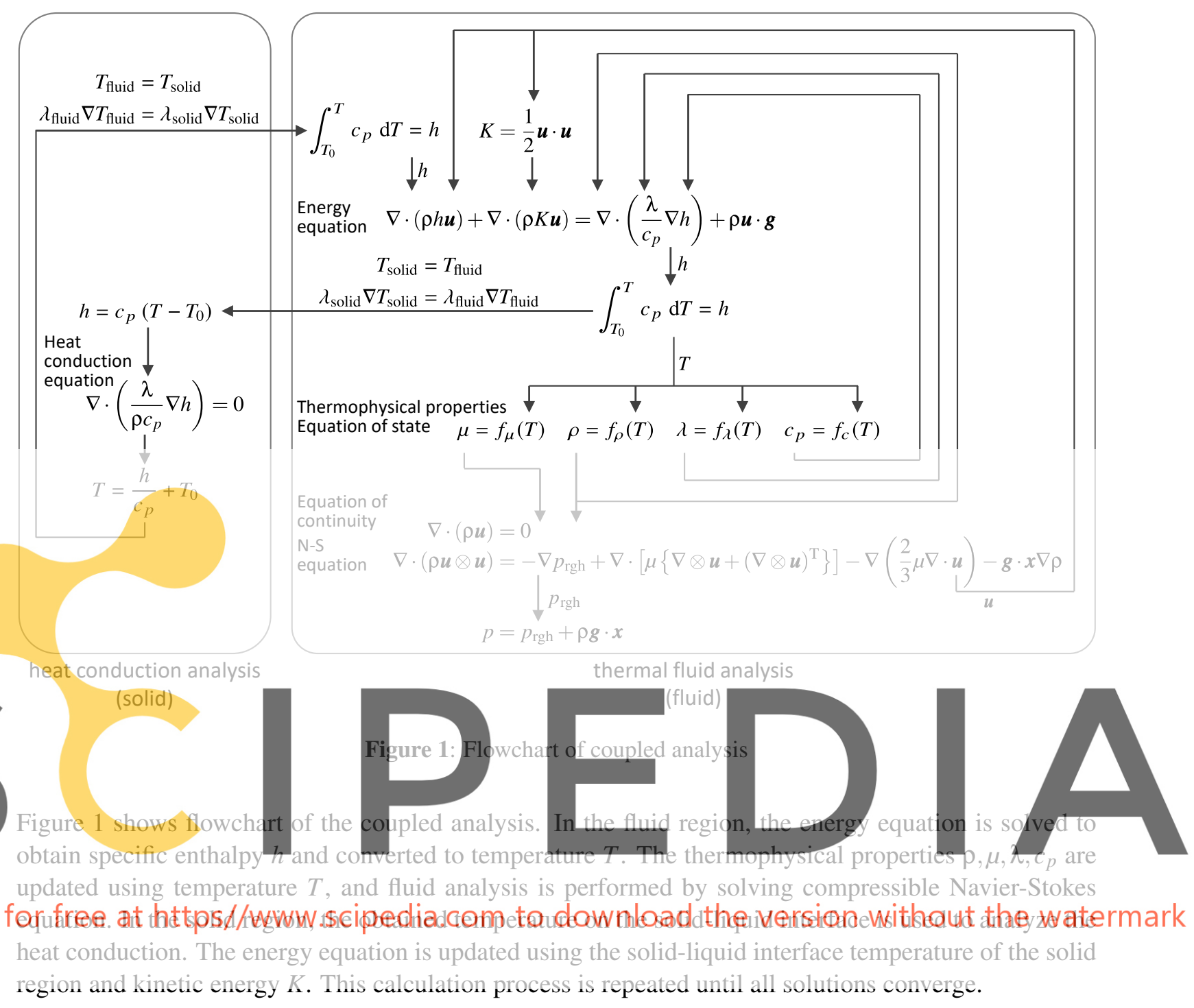

In this study, the standard solver chtMultiRegionFoam of OpenFOAM v7 [2] is used. In order to compare the analysis results, the thermal fluid analysis of the internal fluid using buoyantSimpleFoam and the unsteady heat transfer analysis of the vessel body using the laplacianFoam are also performed to compare the analysis results.

\section{FINITE VOLUME MODEL AND ANALYSIS CONDITIONS}

\subsection{Construction of 3D finite volume model}

In this study, 3-dimensional (3D) solid model shown in Fig. 2 was constructed using a 3D CAD software based on the configuration drawing of the actual reactor. Although there are various structure parts such as pressure sensing tubes inside the actual reactor, in this study, the analysis domain is from the bottom of the vessel to the height of $5 \mathrm{~m}$ in order to focus on the thermal flow phenomena at the bottom of the vessel. 




(a) without partition


(b) with partition
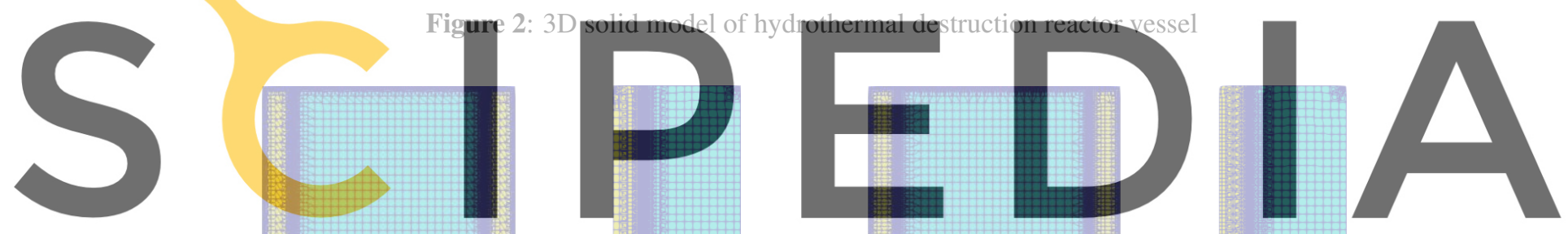

Register for free at https//www.scipedia.com to download the version without the watermark



(a-1) symmetrical plane (a) without partition total $7,457,054$ cells



(b-1) symmetrical plane (b-2) cross section (b) with partition, total $20,299,821$ cells

Figure 3: Finite volume mesh 


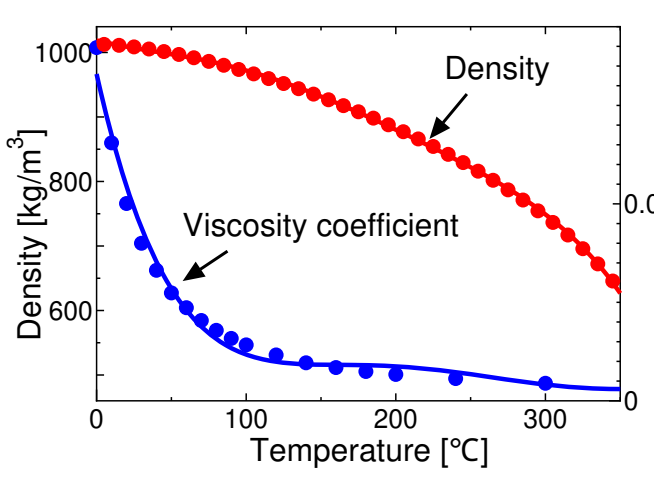

(a) density $\rho$ and viscosity coefficient $\mu$



(b) heat conductivity $\lambda$ and specific heat $c_{p}$

Figure 4: Temperature dependent thermophysical properties of fluid

Table 1: Thermophysical properties of inconel alloy $\left(\mathrm{NCF} 690,20^{\circ} \mathrm{C}\right)$

\begin{tabular}{lc}
\hline Density $\rho$ & $8200 \mathrm{~kg} / \mathrm{m}^{3}$ \\
Specific heat $c_{p}$ & $450 \mathrm{~J} /(\mathrm{kg} \mathrm{K})$ \\
Thermal conductivity $\lambda$ & $12 \mathrm{~W} /(\mathrm{m} \mathrm{K})$ \\
\hline
\end{tabular}

Table 2: Boundary conditions


Side wall of treatment fluid nozzle Oxygen inlet

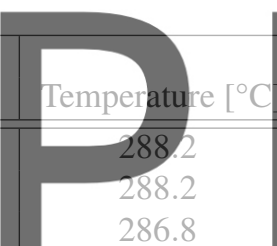

286.8

19.1

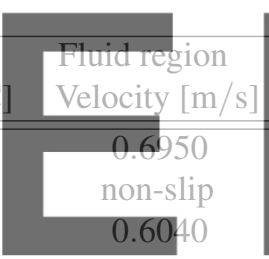

non-slip

0.0467
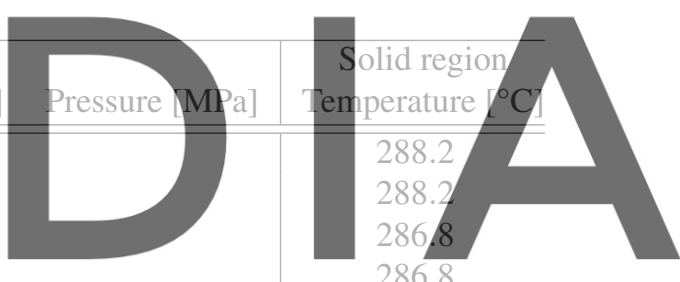

286.8

19.1

\begin{tabular}{l|cccc} 
Bottom supply inlet & 336.1 & 0.0176 & 26.5 & 336.1 \\
Outlet (top surface) & 369.9 & uniform & - & 369.9 \\
Outer wall of vessel & - & - & & insulation \\
Inner wall of vessel & heat transfer & non-slip & & heat transfer \\
Bottom partition & heat transfer & non-slip & & heat transfer \\
\hline
\end{tabular}

The analysis of half model of the reactor vessel is carried out considering symmetry structure. Two types of FVA models are constructed with and without the bottom partition to compare results.

Unstructured FVA mesh is generated using a standard meshing utility snappyHexMesh of the OpenFOAM based on 3D geometry data in the standard triangulated language (STL) format of the 3D solid vessel models. Figure 3 shows FVA mesh. In the figure, yellow and light blue cells represent solid and fluid regions, respectively. 


\subsection{Thermophysical properties and boundary conditions}

In order to express temperature dependence of the thermophysical properties of the internal fluid, density $\rho\left[\mathrm{kg} / \mathrm{m}^{3}\right]$, viscosity $\mu[\mathrm{Pas}]$, thermal conductivity $\lambda[\mathrm{W} /(\mathrm{mK})]$, and specific heat $c_{p}[\mathrm{~J} /(\mathrm{kg} \mathrm{K})]$ are approximated by polynomial equations of temperature $T[\mathrm{~K}]$,

$$
f(T)=\sum_{i=0}^{N} a_{i} T^{i}
$$

as shown in Fig. 4, where $N$ is degree of the polynomial, $a_{0}, a_{1}, \ldots, a_{N}$ are coefficients and are calculated by least squares approximation. For the heat conduction analysis of the vessel body, temperature independent thermophysical properties of inconel (NCF690) are shown in Table 1.

Boundary conditions of multi-regionally coupled analysis are summarized in Table 2. At each inlet of the fluid region, temperature and flow velocity are set based on the measured values during actual operation of the reactor. Since the oxygen nozzle has a double circular structure and is constantly cooled by cooling water, temperature on the side wall of the nozzle is set to $19.1^{\circ} \mathrm{C}$. At the outlet, which is the top surface of the FV models, pressure is set to $26.5 \mathrm{MPa}$ due to the measured value inside the actual vessel, and the uniform velocity outlet condition is configured. In addition, the outlet temperature is set to $369.9^{\circ} \mathrm{C}$, which is also the measured value, to simulate the heat generated by the chemical reaction in the hydrothermal oxidative decomposition of PCBs. On the outer wall of the vessel of solid region, heat transfer is insulated. The conjugate heat transfer is considered on the solid-liquid interface that are inner

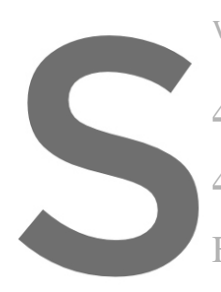
wall of vessel and surfaces of bottom partition.

4 RESULTS AND DIS

4.1 Temperature distribution

Figure 5 shows a comparison of the temperature di
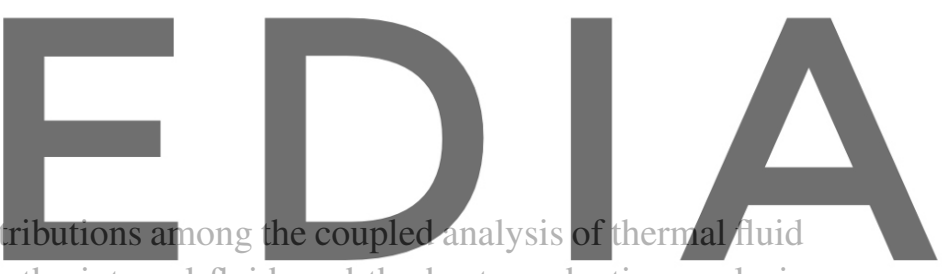

and heat conduction, the thermal fluid analysis for the internal fluid, and the heat conduction analysis

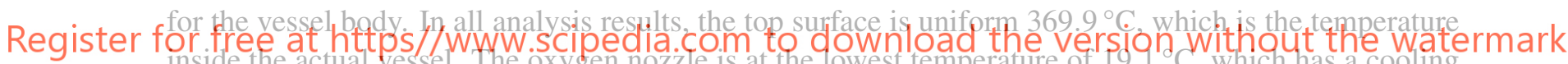
effect on bottom of the vessel.

Comparing the temperature distribution results at the bottom of the vessel, the results of the thermal fluid analysis for the internal fluid is the lowest temperature, and the heat conduction analysis for the vessel body is the highest temperature. In the thermal fluid analysis for the internal fluid, since the solid-liquid interface acts heat insulation, the temperature is reduced by the descent of the fluid cooled by the oxygen nozzle. The heat conduction analysis of the vessel body retains the hot water temperature at the inlet of the bottom. In contrast, the results of the coupled analysis are distributed between the temperatures of the other two results because of the heat transfer on the solid-liquid interface.

As shown in Fig. 5 (b), since heat conduction does not occur across partition in the thermal fluid analysis, the temperature differs significantly between top and bottom of the partition. The results of the coupled analysis show that the temperature in the lower region of the bottom partition is lower than without the partition because advection is blocked by the bottom partition.

Figure 6 shows comparisons of temperature distributions on the solid-liquid interface along a path from the center of the vessel bottom to the side wall on the symmetry plane opposite the oxygen nozzle as 




Figure 5: Temperature distribution 

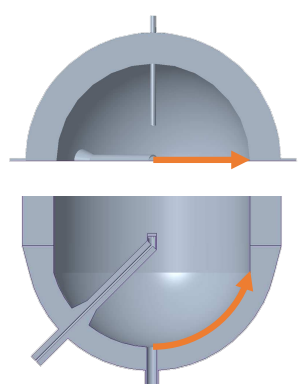

(a) comparison location

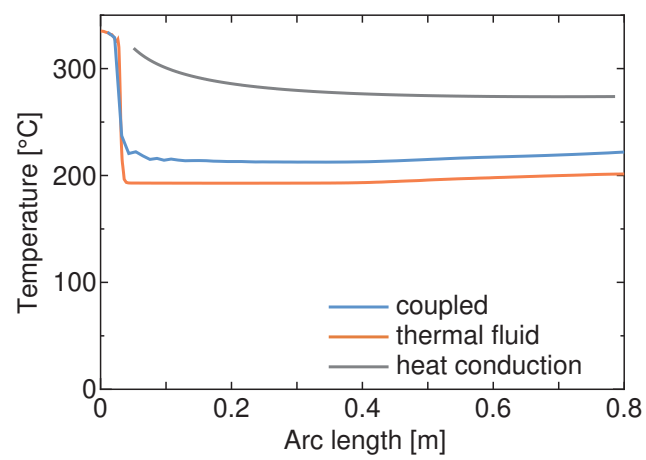

(b) without partition

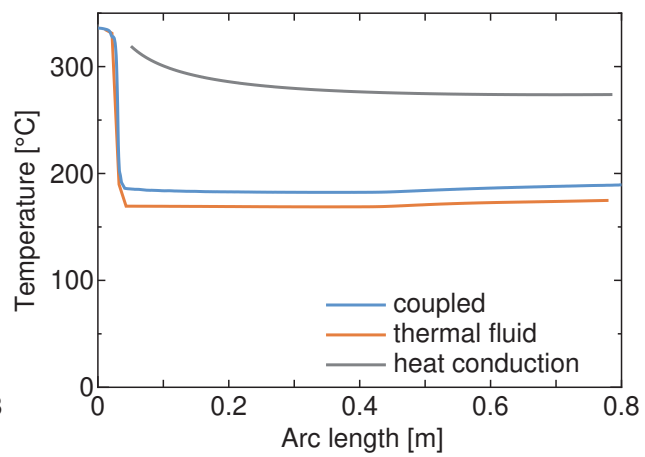

(c) with partition

Figure 6: Temperature comparison at bottom of the vessel
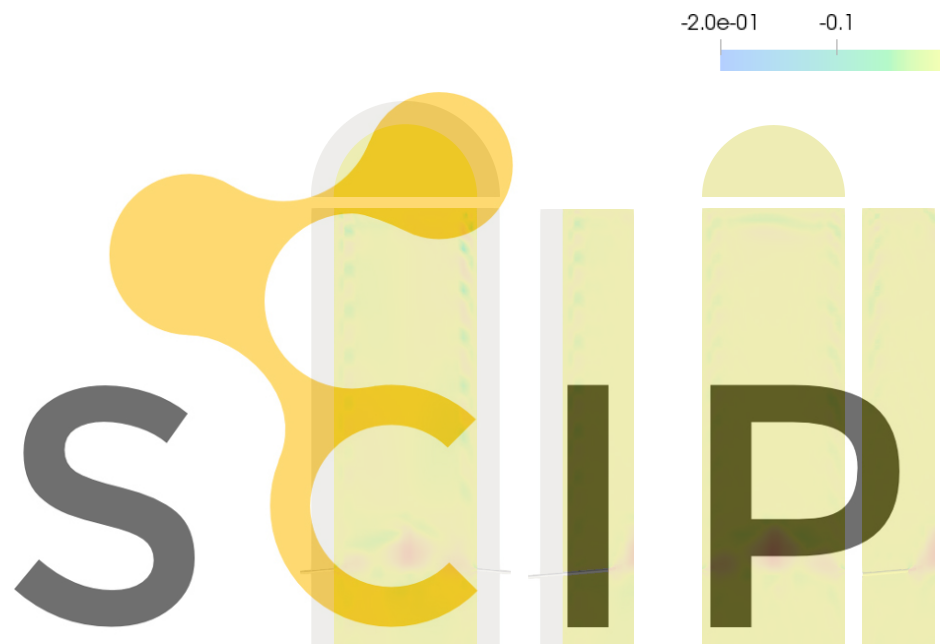

$0.1 \quad 2.00-01$

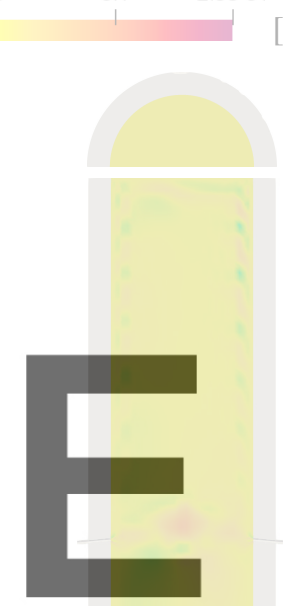

$[\mathrm{m} / \mathrm{s}]$

Register for free at https//www.scipedia.com to download the version without the watermark

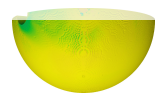

(a-1) coupled

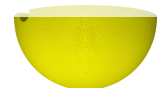

(a-2) thermal fluid

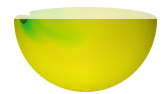

(b-1) coupled



(b-2) thermal fluid

(a) without partition

(b) with partition

Figure 7: Velocity distribution in the vertical direction

$\begin{array}{ccccc}-2.0 e-01 & -0.1 & 0 & 0.1 & 2.0 e-01\end{array}$

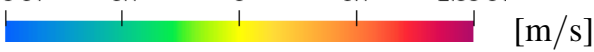

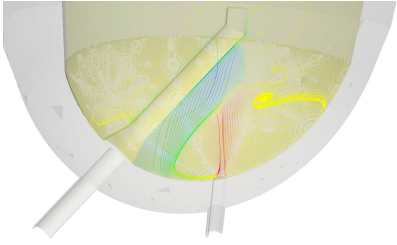

(a-1) coupled

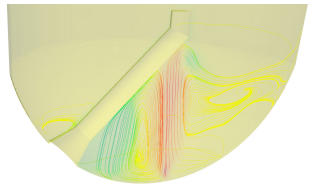

(a-2) thermal fluid

(a) without partition



(b-1) coupled



(b-2) thermal fluid

(b) with partition

Figure 8: Stream line 
indicated by the orange line in Fig. 6 (a). With or without the bottom partition, the heat conduction analysis results of the vessel show higher temperature than the others because there is no advection by liquid oxygen and the fluid cooled by the oxygen nozzle. The results of the coupled analysis are higher than those of the thermal fluid analysis because of heat conduction in the vessel body.

\subsection{Velocity distribution}

The velocity distribution in the vertical direction which is height direction of the vessel is shown in Fig. 7 in comparison with the coupled analysis and the thermal fluid analysis. The outlet on the top surface has a uniform flow velocity, which is $0.00129 \mathrm{~m} / \mathrm{s}$ in the vertical direction. The actual average flow velocity $3 \mathrm{~m} / \mathrm{h}=0.00083 \mathrm{~m} / \mathrm{s}$ for the entire reactor of height $15 \mathrm{~m}$ with an in-vessel processing time of $5 \mathrm{~h}$ is similar order as the result around the top surface. In the bottom of the vessel, the fluid is cooled by the oxygen nozzles and is descended, and the hot water from the bottom inlet flows upward in both analysis results. In the coupled analysis, the downward flow is more extensive because the temperature between the oxygen nozzle and bottom of the vessel is larger than in the thermal fluid analysis.

Streamlines at the bottom of the vessel are shown in Fig. 8. The streamline color indicates the vertical flow velocity. The bottom supply inlet flow rises hot water flowing but the fluid is fallen down by the oxygen nozzle cooling, then causing circulation. In coupled analysis, the downward flow is larger than the thermal fluid analysis.

\section{CONCLUSIONS}



In this study, multi-regiona

analysis for the vessel

considering the heat tra

inside the reactor is introct

actual operation. The effec
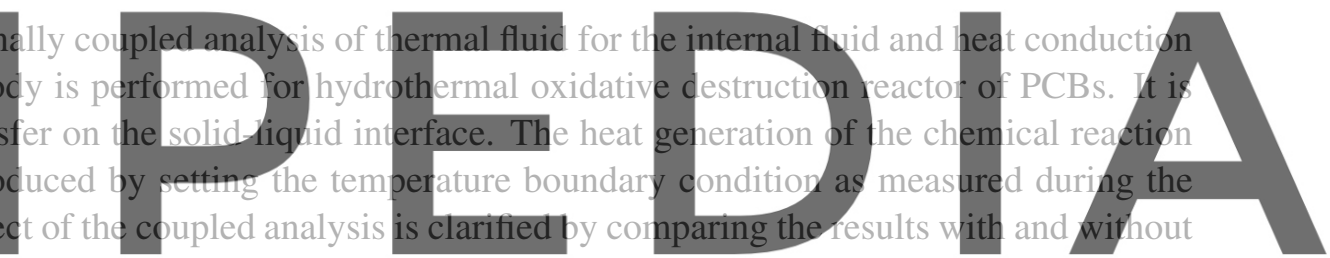

the bottom partition, and the coupled analysis with the uncoupled analyses.



The authors would like to acknowledge providing actual data of the hydrothermal oxidative destruction reactors and the financial support of the Tokyo PCB Waste Treatment Facility, Japan Environmental Storage \& Safety Corporation (JESCO).

\section{REFERENCES}

[1] Maeda, T., Ueda, K., Fukusumi, T., Shinoda K. and Tateishi, M. Treatment of PCB by Hydrothermal Decomposition. Mitsubishi Heavy Industries Technical Review, (2001) 38-1: 32-35 (in Japanese).

[2] OpenFOAM (Free CFD Software) The OpenFOAM Foundation, https://openfoam.org/ 